\title{
Impact of system parameter selection on radar sensor performance in automotive applications
}

\author{
H.-L. Blöecher ${ }^{1}$, M. Andres ${ }^{2}$, C. Fischer ${ }^{1}$, A. Sailer ${ }^{2}$, M. Goppelt ${ }^{2}$, and J. Dickmann ${ }^{1}$ \\ ${ }^{1}$ Daimler AG, Group Research and Advanced Engineering, 89081 Ulm, Germany \\ ${ }^{2}$ University Ulm, Institute of Microwave Techniques, 89081 Ulm, Germany \\ Correspondence to: H.-L. Blöcher (hans-ludwig.bloecher@daimler.com)
}

\begin{abstract}
The paper deals with the investigation of relevant boundary conditions to be considered in order to operate $77 / 79 \mathrm{GHz}$ narrow and ultra wide band automotive radar sensors in the automotive platform and the automotive environment.
\end{abstract}

\section{Introduction}

Millimeterwave radar is a vital component of present and future automotive environmental sensing systems. Information given by these systems is essential for the realization of automotive safety- and driver assistance functions. Automotive radar sensors operating in the $77 / 79 \mathrm{GHz}$ band will, due to frequency regulation constraints, offer the only remaining option to operate at UWB bandwidth $(>500 \mathrm{MHz})$. Moreover, due to the decreasing sensor dimensions with increasing frequency, W-band radar will offer increased angular resolution using antennas with moderate aperture dimensions.

The frequency range $77 \mathrm{GHz}$ to $81 \mathrm{GHz}(79 \mathrm{GHz})$, has been defined by the European Commission in 2004 as the frequency allocation for automotive short range radar systems. The initial intention and objective of this $79 \mathrm{GHz}$ project is to establish and speed up the worldwide harmonised frequency allocation for vehicular radars in that frequency range. The $79 \mathrm{GHz}$ automotive frequency band, once available worldwide, will promote and accelerate the transition from the currently used $24 \mathrm{GHz}$ band, which is an intermediate regulatory solution, to the $79 \mathrm{GHz}$ band.

As a consequence of the $24 / 79 \mathrm{GHz}$ frequency allocation for UWB automotive radar in Europe by 2005, industry had to show honest efforts to develop and provide a cost efficient technology platform at the $77 / 79 \mathrm{GHz}$ band. Thus, the Ger- man BMBF public funded projects KOKON (2004-2007) and RoCC (2008-2011) were established. Within the BMBF public funded project RoCC - "radar-on-chip for cars", the necessary cost efficient $\mathrm{SiGe}$ based technology for the realization of W-band radar is developed. Furthermore, relevant technical constraints to be considered for operation of these sensors are investigated and the consequences with respect to sensor specifications are derived. Within the paper, some very essential constraints are discussed:

- Covered/buried placement and packaging of $\mathrm{W}$ band radar within the automotive platform, which leads to the need to design car body parts as radomes.

- Necessary operational bandwidth, derived from relevant applications and used for the realization of adequate range and angular resolution.

- Car to car interference and possible countermeasures, in particular with respect to realization of safety functions and proposed market penetration rates of automotive radar.

Finally, the potential of DBF/DoA approaches to efficiently contribute to the solution of these problems will be discussed.

\section{Selection of application driven radar parameters}

Due to diverse urban scenarios, radar sensors have to cope with different target types, like pedestrians, motorbikes, cars and trucks. Hence, urban scenarios require highly sophisticated radar sensor performance. High range and angular resolution are essential to detect two closely neighbouring targets with unequal radar cross sections and the same velocity. For example, a pedestrian waiting in between two parked cars and intending to cross the street, requires high range and 

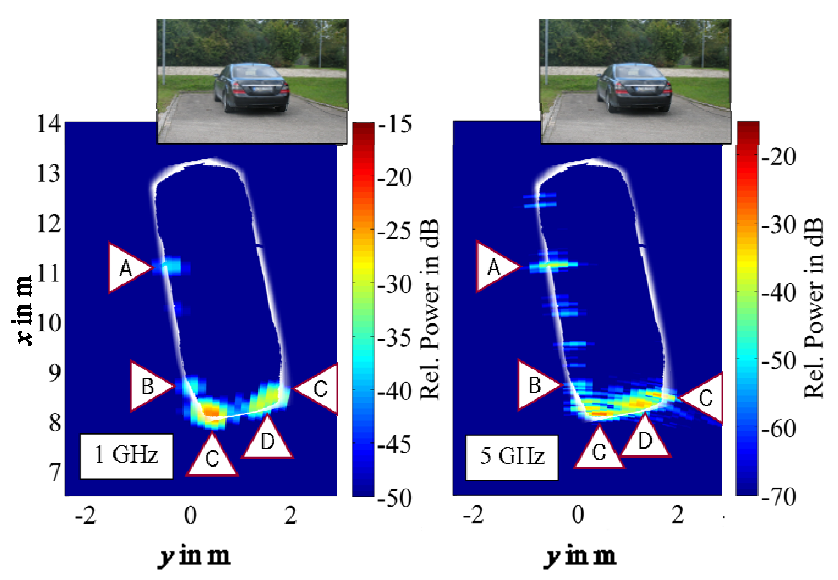

Fig. 1. Measurement result of the contour determination of a car using a bandwidth of $1 \mathrm{GHz}$ (left) and $5 \mathrm{GHz}$ (right) at a carrier frequency of $77 \mathrm{GHz}$ and an angular resolution of $1^{\circ}$.

angular resolution of the radar sensor. To estimate the time to collision, the length, width and orientation of the car and the exact position of the pedestrian have to be determined. Having preliminary knowledge of the main locations of the scattering centers enables the evaluation of the situation to be more precise. Strong reflection regions of a car are located at the rearview mirror, transition between running surface and asphalt, light units and the license plate (Andres, 2011).

To extract the contour of a car, the required bandwidth and angular resolution have to be determined. In Fig. 1, a $77 \mathrm{GHz}$ FMCW radar frontend used in (Feil, 2010) with a high directive antenna was attached to a mechanical scanning unit. The radar sensor was equipped with a double folded reflect array antenna with a half power beam width (HPBW) of approximately $1^{\circ}$ (two way pattern). Therefore, the angular stepping size of the mechanical scanning unit was adjusted to $1^{\circ}$. The radar sensor was placed behind the car at the origin of the coordinate system at a distance of $8 \mathrm{~m}$. In Fig. 1 left a bandwidth of $1 \mathrm{GHz}$ and in Fig. 1 right a bandwidth of $5 \mathrm{GHz}$ was adjusted to the FMCW radar. Comparing these two images the increase of the bandwidth enables a more precise extraction of the contour of the vehicle. However, the main scattering centers like the left rearview mirror (A), the transition between running surface of the left wheel and asphalt (B), the rear light units (C) and the license plate (D) could be extracted even at the bandwidth of $1 \mathrm{GHz}$.

The angular resolution of the radar sensor has an impact on the contour determination of the target as well. To analyze this influence two different antenna types were used to scan the same measurement scenario. In Fig. 2 left and right the car was located at a distance of $10 \mathrm{~m}$ with a gear angle of $30^{\circ}$. In Fig. 2 left, the previous reflected fold array antenna with a HPBW of $1^{\circ}$ was applied on the radar sensor and in Fig. 2 right a lens horn antenna with a HPBW of approximately $3^{\circ}$ (two way) was attached. For both measurements a bandwidth

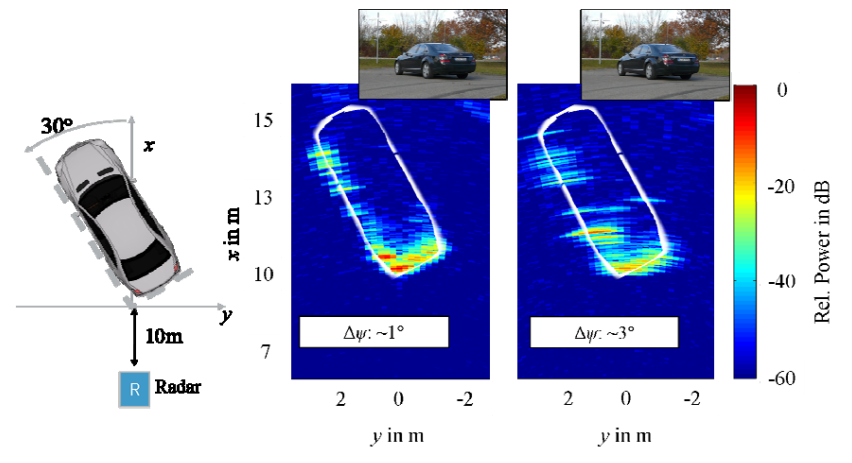

Fig. 2. Measurement result of the contour determination of a car using a bandwidth of $6 \mathrm{GHz}$ and an angular resolution of $\sim 1^{\circ}$ (left) and $\sim 3^{\circ}$ at a carrier frequency of $77 \mathrm{GHz}$.

of $6 \mathrm{GHz}$ was adjusted. The measurement results show that by reducing the angular resolution capability from $1^{\circ}$ to $3^{\circ}$, the uncertainty of the width of the car increases and hence, the determination of orientation is more difficult. Furthermore, the interference between two neighbouring scattering centers increases. This effect can cause a destructive interference and hence a loss of the detectable scattering center.

In conclusion, using a bandwidth larger than $1 \mathrm{GHz}$, and an angular resolution better than $1^{\circ}$, enables estimation of the contour of a vehicle. This leads to an improved detection of the target, resulting in higher accuracy for the post processed tracking.

\section{Lateral resolution and flexible antenna pattern adaption}

The number of people living in cities and also the number of cars in the streets is expected to increase significantly within the next years (IAE, 2008). Due to this development the cars and also the sensors used inside the cars need to be adapted to this environment with growing complexity. The requirements for the sensors used in an inner city environment differ largely from those used on highways or motor-ways where the number of cars is small. Also close encounters of similar sensors occur seldom and only for a short time. The inner city scenario is the exact opposite: One encounters many cars and therefore has the necessity to differentiate between these with high reliability. These cars may also be equipped with similar sensors. Therefore interference free coexistence must also be assured.

The challenge to be faced in the inner city scenario is the reliability of the measurements. Resolution of the measurements in range was already covered but also the lateral resolution and accuracy is becoming more and more important. In the environment faced on highways movement is mostly in the range domain. In scenarios with turning cars, tracking of lateral movement also comes into play. In radar sensors lateral accuracy is directly correlated with angular accuracy 

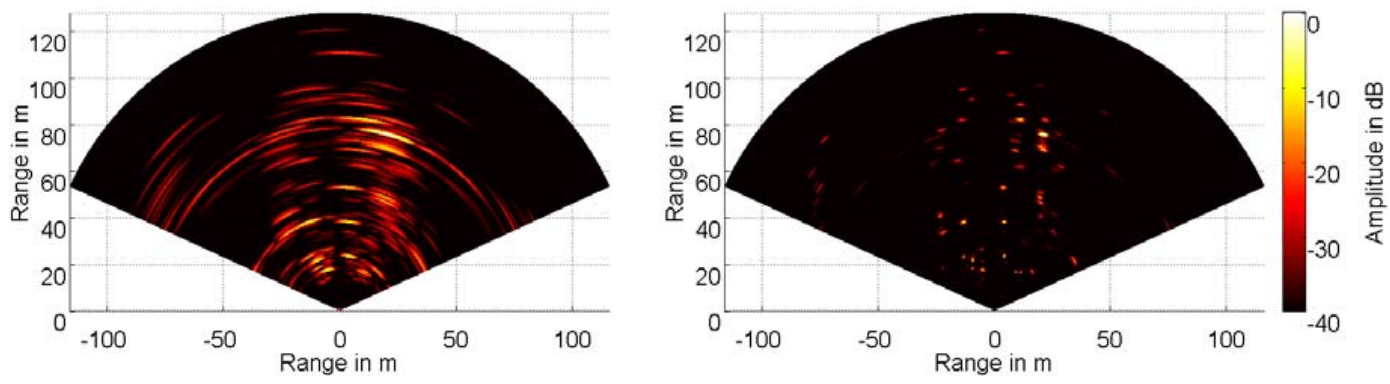

Fig. 3. Radar plot of a scenario processed with traditional beamforming (left) and beamforming using linear prediction with 5 coefficients (right). Scale is the same in both plots.

which depends on the aperture of the antenna. The aperture is proportional to the antenna's size and its operating frequency. As space is very limited in today's cars, the size of the sensor and therefore the antenna size is limited. To increase the resolution of a sensor one can either move to a higher frequency, which is limited by currently available technology, or use Digital Beamforming in combination with super resolution techniques (Feil, 2007). These techniques trade antenna space for computational complexity.

Figure 3 gives an example for the potential of super resolution techniques. In this case the method of linear prediction with five coefficients was applied as described in detail e.g. in Mayer (2008). One can see that with traditional beamforming many scatterers are blurred. By applying additional signal processing this blur can be reduced significantly and contours become more clearly visible. Another possible advantage of this high angular resolution is that interferers may be suppressed with higher selectivity. The necessary steps for this adaptive beamshaping will be the subject of further research.

\section{Sensor - sensor interference and interference mitigation}

Daimler's activities in the RoCC project with respect to sensor interference and interference mitigation aim at understanding interference mechanisms as well as testing and evaluating countermeasures (interference mitigation methods). For this purpose a radar simulator was created, analytic calculations were performed, and measurements with different radar frontends were conducted in the laboratory and with a vehicle.

For example, a vehicle with a radar frontend using FMCW modulation was moved towards a stationary frontend emitting a $\mathrm{CW}$ signal. The effect of the interfering $\mathrm{CW}$ signal at $77.5 \mathrm{GHz}$ intersecting the $\mathrm{FMCW}$ frequency ramp (with a rise time of $2 \mathrm{~ms}$ from $77 \mathrm{GHz}$ to $78 \mathrm{GHz}$ ) can be seen in Fig. 4 in the time domain (upper diagram) and in the frequency domain (lower diagram). The interference creates a wideband signal at the IF port of the mixer which reduces the sensitivity of the radar (Goppelt, 2011).
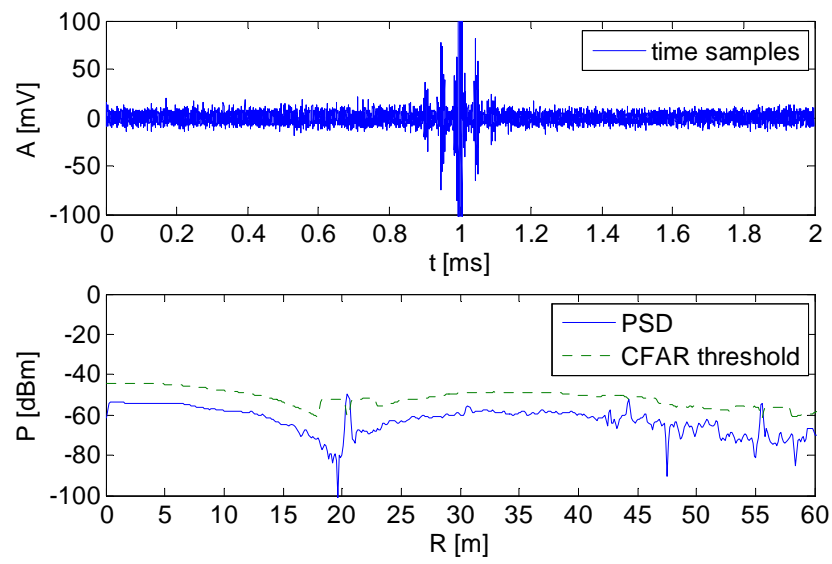

Fig. 4. FMCW radar signal with interference from a CW interferer. Upper diagram: the time samples taken by the analog-to-digital converter. Lower diagram: the signal in the frequency domain (power spectral density) along with the CFAR threshold.

One possible way to reduce the effect of the interference is to detect and weight (multiply) the affected samples with the following weighting function:

$w(i)=\left(1-0.5 \cdot\left(1-\cos \left(\frac{2 \cdot \pi \cdot\left(i-i_{1}\right)}{i_{2}-i_{1}}\right)\right)\right)^{4}$,

with

$i_{1} \leq i \leq i_{2}$.

It can be seen in Fig. 5 that this countermeasure significantly reduces the interference, resulting in a greatly improved sensitivity. Furthermore, it is easy to implement, since it consists purely of digitally processing the time samples without the need to modify the frontend.

\section{Covered platform integration of $77 / 79 \mathrm{GHz}$ automotive radar sensors}

Within the car, short range radar (SRR) sensors are typically mounted invisibly behind painted bumpers or other layered 

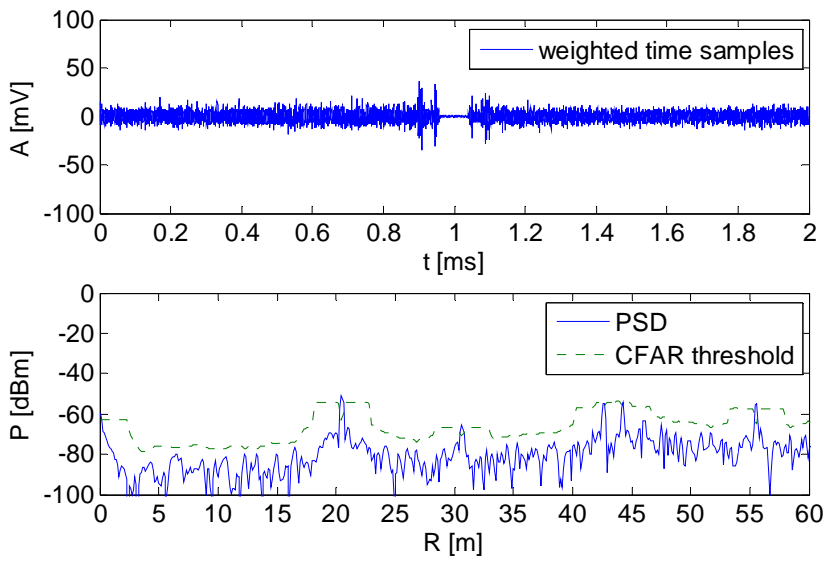

Fig. 5. The FMCW radar signal after applying the countermeasure (weighting of the time samples).

components. Any cover of an antenna of a radar sensor has to be carefully designed, in order to avoid performance degradation due to transmission losses, reflections, and edge effects. This is a crucial issue concerning sensor systems working in the frequency range from $76 \mathrm{GHz}$ to $81 \mathrm{GHz}$ even more than for lower operating frequencies. Bumpers and other kinds of components mounted on a vehicle's front or rear-end have to be considered as radome structures. For the desired frequency range, their thickness is commonly in the order of not more than a couple of wavelengths.

The OEMs among the project partners, Daimler and BMW, have to consider a wide range of sensor packaging aspects in RoCC. Special attention has to be turned on the impact of the necessary frequency bandwidth around $79 \mathrm{GHz}$. This parameter is influenced by the electromagnetic characteristics of bulk material and painting (permittivity and loss tangent), manufacturing tolerances, multiple paintings (especially repair paintings) and covering of radomes with water, snow, ice, dust or salt, etc.

In Bloecher (2009a, b), first results concerning sensor integration behind painted bumpers and particular LRR radomes are depicted. Extensive studies of manufacturing tolerances and the composition of substrate materials as well as paint were carried out by Daimler within the project RoCC. As an exemplary result, Fig. 6 shows the transmission $|t|$ and the reflection $|r|$ through a bumper with metallic paint. The simulation was carried out with a multi-layer model implemented in Matlab. For the ideal case of properly chosen substrate thickness $d_{\text {sub }}=3.44 \mathrm{~mm}$, the reflection $|r|$ is found to be better than $-20 \mathrm{~dB}$ for the whole SRR frequency band of 77 to $81 \mathrm{GHz}$. Introducing a variation of the equivalent permittivity of the paint would shift the desired minimum of $|r|$ to lower or higher frequencies. Nevertheless, the transmission $|t|$ stays approximately constant.

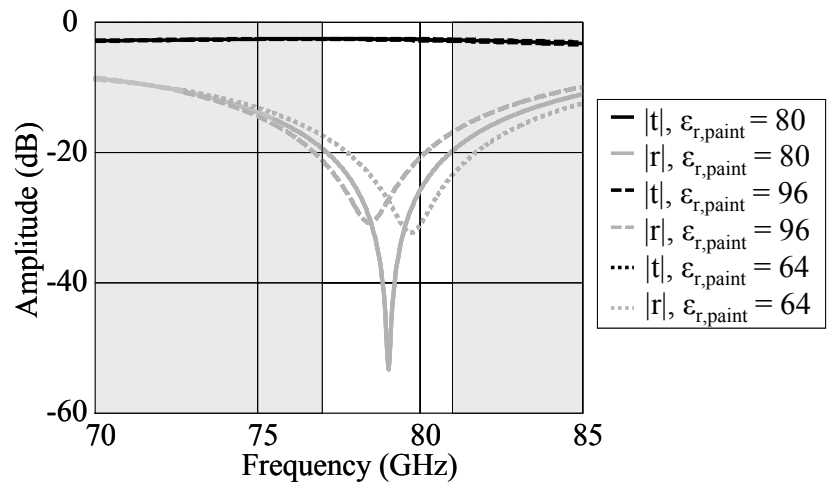

Fig. 6. Simulation of transmission $|t|$ and reflection $|r|$ through a bumper with metallic paint. Variation of the permittivity of the paint $\varepsilon_{r, \text { paint }}$ shifts the reflection minimum.

A radome that is robust against variation of the material parameters and thickness of substrate and paint has to provide sufficient transmission bandwidth. As a consequence, variations of dedicated scale would not seriously affect the radar's performance. Considering manufacturing tolerances, varying material combinations and required bandwidth, thickness optimization of the substrate material was found to be a good compromise between RF performance, costs and effort. Nevertheless, this compromise is strongly dependent on specific sensor requirements and the specific car model's needs. In the case where thickness matching does not provide sufficient RF performance, one could apply broad band design methods including frequency selective surface structures or inductive structures on the back side of the bumper as shown in (Pfeiffer, 2009). In conclusion, meeting the desired RF performance always means balancing effort in RF engineering with keeping dimensional accuracy and material composition.

\section{Conclusions}

Automotive radar is an essential component of present and future automotive environmental sensing systems used for the realization of automotive active safety and comfort functions. Due to regulatory reasons as well as the ability to use the same technology platform for long-, mid- and short range automotive radar, the $77 / 79 \mathrm{GHz}$ frequency band is the future choice. The development of a low cost and robust SiGe based technology platform has been done within the BMBF public funded projects KOKON and RoCC. Beneath the availability of an adequate technology platform, further technical and operational requirements have to be fulfilled to operate the sensor within the automotive platform and the environment. Within the project's methods, solutions with respect to platform integration, sensor to sensor interference and application driven sensor parameter selection and have been developed and characterized successfully. These results are 
to be transferred into series development of the radar sensors. Furthermore, DBF/DoA/adaptive beamforming algorithms could potentially be used to obtain improved angular resolution with limited physical antenna dimensions. The flexibility of the adaptive antenna pattern will be used for suppression of interfering signals to keep and improve, for example, the radar sensitivity.

Acknowledgements. The authors wish to thank the German Federal Ministry of Education and Research (BMBF) for the funding of the RoCC project. The authors also wish to thank Stacey Rukezo (currently with Daimler AG) for proofreading the manuscript.

\section{References}

Andres, M., Feil, P., Menzel, W., Bloecher, H.-L., and Dickmann, J.: Analysis of automobile scattering center locations by SAR measurements, Proc. of Radar Conference 2011, Kansas City, USA, Mai, 2011.

Bloecher, H.-L., Sailer, A., Rollmann, G., and Dickmann, J.: 79 GHz UWB automotive short range radar - Spectrum allocation and technology trends, Adv. Radio Sci., 7, 61-65, 2009,

http://www.adv-radio-sci.net/7/61/2009/.
Bloecher, H.-L., Sailer, A., Andres, M., Goppelt, M., and Dickmann, J.: Trends in Development of SiGe based 76-81 GHz Automotive Radar, IRS 2009 - International Radar Symposium, Hamburg, 2009.

Feil, P., Mayer, W., Menzel, W., and Guehl, R.: Various Cross Range Resolution Techniques Applied to an Eight Channel 77 GHz Sensor, EuRAD 2007, Munich, Germany, 59-62, 2007.

Feil, P., Mayer, W., Kraus, T., Migliaccio, C., and Menzel, W.: Industrial and Security Applications of Imaging Millimeter Wave Sensors, Technisches Messen, July-August, 2010.

Goppelt, M., Blöcher, H.-L., and Menzel, W.: Analytical investigation of mutual interference between automotive FMCW radar sensors, Proc. of GEMIC 2011, Darmstadt, Germany, 2011.

International Energy Agency (IEA): World Energy Outlook 2008, 2008.

Mayer, W.: Abbildender Radarsensor mit sendeseitig geschalteter Gruppenantenne, Dissertation, Ulm, 2008.

Pfeiffer, F. and Biebl, E. M.: Inductive Compensation of High-Permittivity Coatings on Automobile Long-Range Radar Radomes, IEEE T. Microw. Theory., 57, 2627-2632, November, 2009 . 